\title{
Revisiting Proportional Fairness: Anonymity Among Users in Interference Coupled Wireless Systems
}

\author{
Holger Boche, Senior Member, IEEE, and Siddharth Naik
}

\begin{abstract}
The paper revisits the problem of proportional fairness in interference coupled wireless systems. It models interference coupling in wireless systems based on an interference function framework through a set of axioms (introduced by Yates in 1995). It utilizes the collective choice function to represent resource allocation strategies and an axiomatic framework to emulate certain desirable properties of resource allocation strategies. We introduce the axiom of equal priority in the power domain (and in the interference domain) and motivate it as interference coordination fairness. We consider this as an anonymity among the users, from the perspective of a central controller, e.g. a base station or an operator. We show that the proportional fair resource allocation strategy is anonymous to the identity of the users at the signal processing layer. Such an anonymity is relevant to obtain interference coordination fairness in iterative resource allocation strategies frequently encountered in wireless systems.
\end{abstract}

Index Terms-Proportional fairness, anonymity among users, interference coupled wireless systems.

\section{INTRODUCTION}

$\mathbf{T}$ HE topic of proportional fairness has been well motivated and analyzed in literature. The work [1] analyzes a problem similar to the one considered in our paper. In [1] the problem of optimal flow control in a multiclass telecommunications environment where each user desires to optimize its performance while being fair to the other users is considered. They suggest the Nash bargaining solution (NBS) as a suitable candidate for this problem. This work has been extended by [2], who analyze stability and fairness of two classes of rate control algorithms for communication networks. They motivate proportional fairness from a stability perspective and generalize the problem for routing control. The papers [3]-[6] are some of the more recent work on proportional fairness in wireless systems. Our list is by no means exhaustive. A further list of references can be found at [7].

Our paper revisits the problem of proportional fairness resource allocation strategy. Our paper utilizes the collective choice function (CCF) to represent resource allocation strategies and an axiomatic framework to capture certain desirable properties of resource allocation strategies. We motivate that

Paper approved by T.-S. P. Yum, the Editor for Packet Access and Switching of the IEEE Communications Society. Manuscript received July 8, 2009; revised January 4, 2010, April 20, 2010, and June 17, 2010.

$\mathrm{H}$. Boche is with the Technical University of Berlin, Heinrich Hertz Institute, Einsteinufer 25, 10587 Berlin, Germany (e-mail: holger.boche@mk.tuberlin.de, boche@hhi.fhg.de).

S. Naik is with the Technical University of Berlin, Heinrich Hertz Chair of Mobile Communication, Einsteinufer 25, 10587 Berlin, Germany (e-mail: naik@hhi.fraunhofer.de).

This work was partly supported by the Deutsche Telekom SAGa project and the Bundesnetzagentur (German Telecom Regulator).

Digital Object Identifier 10.1109/TCOMM.2010.082710.090376 the proportional fairness resource allocation strategy is the unique fair strategy from a signal processing perspective. We introduce the axiom of equal priority in the power domain (interference domain) to capture the property that a central controller (e.g. operator, base station) is anonymous to the identity of the users while carrying out interference management and coordination in the power domain (interference domain).

We make the following assumption: the central controller can measure and knows perfectly the interference values at the users. Such an assumption is frequently encountered in iterative power control algorithms, where the user has to report back the measured interference to a central controller or if a user has to iterate its power value based on a fed back interference value.

The paper has the following main contribution: Under the stated assumption, our main result proves that the central controller (e.g. operator, base station) is anonymous to the users while doing interference management and coordination in the power domain (interference domain), if and only if it implements the proportional fairness resource allocation strategy. We call this anonymity to user permutation when the interference value is known to the user as interference coordination fairness.

\section{INTERFERENCE COUPLing IN WiRELESS SySTEMS}

We begin this section by presenting the notation used in our paper: Let $\mathcal{I}$ represent interference functions. $K$ is the number of users in the system. $\gamma$ represents a vector, such that (s.t.) $\gamma=\left[\gamma_{1}, \ldots, \gamma_{K}\right]^{T} . \gamma_{k}$ is a scalar for $k \in\{1, \ldots, K\}=$ : $\mathcal{K}$. $\Gamma$ represents a set. Let $\mathcal{Y}^{K}$ represent a family of sets for the $K$ users such that $\Gamma \in \mathcal{Y}^{K}$ and $\Gamma \subset \mathbb{R}_{+}^{K} \cdot \gamma^{(1)} \leq \gamma^{(2)}$ implies that $\gamma_{k}^{(1)} \leq \gamma_{k}^{(2)}, \forall k \in \mathcal{K} ; \gamma^{(1)}<\gamma^{(2)}$ implies that $\gamma_{k}^{(1)}<\gamma_{k}^{(2)}, \forall k \in \mathcal{K}$. Similarly for $\geq$ and $>$. Equipped with these definitions and notations, we introduce the interference function framework below.

The signal-to-interference (plus noise) ratio (SINR) is an important measure for user performance in wireless systems. Many other performance measures have a direct relationship with SINR. Consider $K$ users with transmit powers $\boldsymbol{p}=$ $\left[p_{1}, \ldots, p_{K}\right]^{T}$ and $\mathcal{K}:=\{1, \ldots, K\}$. The noise power at each receiver is $\sigma^{2}$. Hence the SINR at each receiver depends on the extended power vector $\boldsymbol{p}=\left[\boldsymbol{p}, \sigma^{2}\right]^{T}=\left[p_{1}, \ldots, p_{K}, \sigma^{2}\right]^{T}$. The resulting SINR of user $\bar{k}$ is $\operatorname{SINR}_{k}(\boldsymbol{p})=p_{k} / \mathcal{I}_{k}(\boldsymbol{p})$, where $\mathcal{I}_{k}$ is the interference (plus noise) as a function of $\boldsymbol{p}$. In order to model interference coupling, we shall follow the axiomatic approach proposed in [8], [9] (extended in [10]). Let $\mathcal{P}$ be the 
set of all power vectors. In our paper, we have $\mathcal{P}:=\mathbb{R}_{+}^{K+1}$ unless explicitly mentioned otherwise.

Definition 1. We say that $\mathcal{I}: \mathcal{P} \mapsto \mathbb{R}_{+}$is an interference function, if the following axioms are fulfilled:

$$
\begin{array}{ll}
A 1 & \text { conditional positivity } \mathcal{I}(\underline{\boldsymbol{p}})>0 \text { if } \underline{\boldsymbol{p}}>\mathbf{0} \\
A 2 & \text { scale invariance } \mathcal{I}(\alpha \underline{\boldsymbol{p}})=\alpha \mathcal{I}(\underline{\boldsymbol{p}}) \text { for all } \alpha>0 \\
A 3 \quad & \text { monotonicity } \mathcal{I}(\underline{\boldsymbol{p}}) \geq \mathcal{I}(\underline{\hat{\boldsymbol{p}}}) \text { if } \underline{\boldsymbol{p}} \geq \underline{\hat{\boldsymbol{p}}} \\
A 4 & \text { strict monotonicity } \mathcal{I}(\underline{\boldsymbol{p}})>\mathcal{I}(\underline{\hat{\boldsymbol{p}}}) \text { if } \underline{\boldsymbol{p}} \geq \underline{\hat{\boldsymbol{p}}}, \\
& \\
p_{K+1}>\hat{p}_{K+1} .
\end{array}
$$

Note that we require that $\mathcal{I}(\underline{p})$ is strictly monotonic with respect to the last component $\underline{p}_{K+1}$. An example is $\mathcal{I}(\underline{\boldsymbol{p}})=$ $\boldsymbol{v}^{T} \boldsymbol{p}+\sigma^{2}$ where $\boldsymbol{v} \in \mathbb{R}_{+}^{K}$ is a vector of interference coupling coefficients. The axiomatic framework A1-A4 is connected with the framework of standard interference functions [8]. For the purpose of this paper it is sufficient to be aware that there exists a connection between these two models and the results of this paper are applicable to standard interference functions. The structure of the SINR region depends on the interference coupling in the system. Until now we have focused on interference coupling aspects, where interference is a function of the powers of the various users and noise.

In this paper, "utility" can represent a certain arbitrary performance measure, which depends on the SINR by a strictly monotonic and continuous function $\phi$ defined on $\mathbb{R}_{+}$. The utility of user $k$ is

$$
u_{k}(\underline{\boldsymbol{p}})=\phi_{k}\left(\operatorname{SINR}_{k}(\underline{\boldsymbol{p}})\right)=\phi_{k}\left(\gamma_{k}(\underline{\boldsymbol{p}})\right), k \in \mathcal{K} .
$$

An example of the above case is capacity: $\phi(x)=\log (1+$ $x)$. Related performance indicators, when we would like to minimize the objective function, which is a function of SINR are minimum mean square error (MMSE): $\phi(x)=$ $1 /(1+x)$, BER for coherent M-ASK (amplitude shift keying), equally spaced amplitudes, equally probable symbols and ML (maximum likelihood) detection for the AWGN channel: $\phi(x)=\frac{2(M-1) Q(\sqrt{x})}{\log _{2}(M) M}$, BER for high-SINR approximation of M-PSK (phase shift keying), equally probable symbols (phases), ML detection, with Gray bit mapping: $\phi(x)=$ $\frac{2}{\log _{2} M} Q\left(\sin \left(\frac{\pi}{M}\right) \sqrt{x}\right)$ [11], [12].

We consider a cooperative game theoretic setting, where the users have the possibility of signalling and communicating with the central controller, e.g. base station, operator before choosing their strategies and the resource allocation process. The users could agree or disagree on the resource allocation strategies. Such a framework is traditionally called a bargaining framework. In our paper, we bargain over closed, bounded and comprehensive sets. There are various tradeoffs and dependencies between the users, which are modelled using this interference function framework and we can obtain an operating point for the system. We have a centralized system, which implies user cooperation allowing them to efficiently manage or even combat interference.

We call the feasible utility set $\boldsymbol{\Gamma}$ (or just the utility set) as the set of all feasible SINR vectors $\gamma$, that can be supported for all users by means of power control, with interference being treated as noise. In our paper we focus on utility sets with the following properties:
- $\boldsymbol{\Gamma}$ is a non-empty closed and bounded subset of $\mathbb{R}_{+}^{K}$. We consider a communication system where at least a single user participates ${ }^{1}$, i.e. relint $\boldsymbol{\Gamma} \cap \mathbf{R}_{+}^{K} \neq \emptyset$.

- $\boldsymbol{\Gamma}$ is comprehensive: A set $\boldsymbol{\Gamma} \subset \mathbb{R}_{+}^{K}$ is called downward comprehensive if for all $\gamma^{(1)} \in \Gamma$ and $\gamma^{(2)} \in \mathbb{R}_{+}^{K}$, $\gamma^{(2)} \leq \gamma^{(1)}$ implies $\gamma^{(2)} \in \boldsymbol{\Gamma}$. This may be interpreted as free disposibility of utility. Throughout the paper, by comprehensive we imply downward comprehensive.

The family of all sets with the above properties is denoted as $\mathcal{S}^{K}$. Hence $\mathcal{S}^{K}:=\left\{\boldsymbol{\Gamma} \mid \boldsymbol{\Gamma} \subset \mathbb{R}_{+}^{K}, \boldsymbol{\Gamma}\right.$ is comprehensive, compact\}.

It can be shown that compact, comprehensive sets from $\mathbb{R}_{+}^{K}$ can be written as sub-level sets of interference functions. An example of certain such sets are certain rate regions. The SINR region $\Gamma$ need not be convex, see Example 1 in [13]. Such an analysis of linear interference functions can be extended to $\log$-convex interference functions. Even though the original SINR region is not convex, by an appropriate transformation to the $\log$-domain (with the substitution $\boldsymbol{p}=e^{s}$, which is component-wise exponential), we are still in a position to analyze the proportional fair resource allocation strategy [13].

It is clear that $\mathcal{S}^{K}$ is closed under intersection and union of its elements. Further we denote the family of all convex and strictly convex utility sets by $\mathcal{S}_{\text {convex }}^{K}$ and $\mathcal{S}_{\text {strict_convex }}^{K}$, respectively. A bargaining game for $K$ users is defined as the pair $(\boldsymbol{\Gamma}, \boldsymbol{d})$ where $\boldsymbol{\Gamma} \subset \mathbb{R}_{+}^{K}$ is the utility set and $d \in\left\{\gamma \in \Gamma: \exists \gamma^{\prime}>\gamma, \gamma^{\prime} \in \Gamma\right\}$ is the disagreement point. The disagreement point is the point in the utility set corresponding to the utilities each user receives, if they are unable to reach a solution outcome via the bargaining game. The disagreement point corresponds to the point with the least utilities obtained by the users in the bargaining game. In our paper we choose $\mathbf{0}$ as the disagreement point to simplify the analysis without any loss of generality. $\gamma \in \Gamma$ is a particular utility vector, where $\gamma=\left[\gamma_{1}, \gamma_{2}, \ldots, \gamma_{K}\right]$ and $\gamma_{k}$ is the utility of the $k^{\text {th }}$ user.

\section{Revisiting Proportional Fairness}

As has been observed in Section I, the proportional fairness resource allocation strategy has been investigated exhaustively in economics and wireless communication systems. The characterization of proportional fairness for resource allocation in wireless systems is very important. We shall be discussing proportional fairness as the only resource allocation strategy that provides interference coordination fairness.

We believe that based on physical layer modeling of wireless systems it would be interesting to complement the analysis

\footnotetext{
${ }^{1}$ We define the relative interior of a set $\mathbf{X}$, denoted as relint $\mathbf{X}$, as its interior relative to aff $\mathbf{X}$ :

relint $\mathbf{X}=\{\boldsymbol{x} \in \mathbf{U} \mid B(\boldsymbol{x}, r) \cap$ aff $\mathbf{X} \subseteq \mathbf{X}$ for some $r>0\}$,

where $B(\boldsymbol{x}, r)=\{\boldsymbol{y} \mid\|\boldsymbol{y}-\boldsymbol{x}\| \leq r\}$ is a ball of radius $r$ and center $\boldsymbol{x}$ in the norm $\|\cdot\|$. (Here, $\|\cdot\|$ is any norm; all norms define the same relative interior.) The set of all affine combinations of points in some set $\mathbf{X} \subseteq \mathbb{R}^{K}$
} is called the affine hull of $\mathbf{X}$ and denoted by aff $\mathbf{X}$ :

$$
\begin{array}{r}
\text { aff } \mathbf{X}=\left\{\theta_{1} \boldsymbol{x}^{(1)}+\ldots+\theta_{n} \boldsymbol{x}^{(n)} \mid \boldsymbol{x}^{(1)}, \ldots, \boldsymbol{x}^{(n)} \in \mathbf{X},\right. \\
\left.\theta_{1}+\ldots+\theta_{n}=1\right\} .
\end{array}
$$


of resource allocation strategies in the utility domain to an analysis in the power domain and propose the following abstraction: power $p_{k}$ is a certain fixed number, which the operator chooses for a user $k$ at each time instant; interference $\mathcal{I}_{k}$ is a certain fixed number, which is the interference caused by other users and noise. However, we assume that interference at each user is fixed at a particular time instant and is known by the central controller (e.g. base station, operator).

Such an abstraction of considering power as a fixed number and interference as a fixed number, which is known to the central controller is frequently used in power update algorithms e.g. Foschini's power control algorithm [14], fixed-point iteration [8] and in general any iterative power control algorithm, which recalculates the power treating the interference as a fixed number for the next step of the iteration.

Such an abstraction is relevant for all decentralized or centralized power control resource allocation strategies, where the nodes or users have to do one of the following: reporting back value of measured interference values to the central controller (e.g. base station); calculating its iterate of the power control value based on the interference value provided to it. These serve as a motivational basis for our characterization of proportional fairness. In the next section, we introduce the $\mathrm{CCF}$, which represents a resource allocation strategy. We further introduce the axiomatic framework, to emulate desirable properties of resource allocation strategies.

\section{A. Collective Choice Function and Utility Function}

We propose a general axiomatic framework, which helps understand the trade-offs between user requirements and different solution outcomes by characterizing resource allocation strategies using the CCF [15]. The CCF chooses one point from a certain set, which is the operating point of the resource allocation strategy. Some examples of such a set are a (signalto-interference) SIR region and certain achievable rate regions.

A common approach is to model the CCF along with a set of axioms, also known as axiomatic bargaining theory [16]. A CCF $\Phi$ represents a resource allocation strategy in the wireless scenario. These game theoretic axioms are used to emulate certain desirable and undesirable properties, which the operating point of our resource allocation strategy could possess. Based on the axioms the CCF satisfies, the corresponding resource allocation strategy satisfies different properties and leads to a different operating point in the region, making the axioms quite intuitive and a natural framework to work within. We begin by defining the CCF $\Phi$.

Definition 2. A CCF on the family $\mathcal{S}^{K}$ of sets $\Gamma$, is defined as any function $\Phi: \mathcal{S}^{K} \mapsto \mathbb{R}_{+}^{K}$ such that $\Phi(\boldsymbol{\Gamma}) \in \boldsymbol{\Gamma}, \forall \boldsymbol{\Gamma} \in \mathcal{S}^{K}$.

For emphasis, as it is a crucial point in obtaining an operating point, we would like to point out that $\Phi(\boldsymbol{\Gamma})$, where $\Gamma \in \mathcal{S}^{K}$ is as per Definition 2 single-valued.

Example 1. The symmetric NBS $\Phi_{N B S}(\boldsymbol{\Gamma})$, where $\boldsymbol{\Gamma} \in$ $\mathcal{S}_{\text {convex }}^{K}$ can be obtained by maximizing the product of the utilities as follows:

$$
\Phi_{N B S}(\boldsymbol{\Gamma})=\arg \max _{\boldsymbol{\gamma} \in \boldsymbol{\Gamma}, \boldsymbol{\gamma} \geq \boldsymbol{d}} \prod_{k=1}^{K}\left(\gamma_{k}\right) .
$$

Let $u: \mathbb{R}_{+}^{K} \mapsto \mathbb{R}_{+}$be any function. Let $u$ be a utility function corresponding to a set a users $\mathcal{K}$ who obtain resources from a central controller.

Example 2. Consider the case, when the interference function looks like $\mathcal{I}_{k}(\boldsymbol{p})=\sigma^{2} /\left|h_{k}\right|^{2}$. Let the utility function $u$ be defined as follows: $u(\gamma)=\prod_{k \in \mathcal{K}}\left(\gamma_{k}\right)$, where $\gamma_{k}=$ $\left(\left|h_{k}\right|^{2} / \sigma^{2}\right) p_{k}$, where $\gamma_{k}$ is the SINR of the $k^{\text {th }}$ user. Then, we have that $u(\gamma)=\frac{1}{\sigma^{2}}\left(\prod_{k \in \mathcal{K}}\left|h_{k}\right|^{2}\right)\left(\prod_{k \in \mathcal{K}} p_{k}\right)$. If we want to maximize the function $u(\gamma)$, subject to a total power constraint $P_{\text {total }}$ on the system -the resulting solution in the power domain is $p_{1}=p_{2}=\ldots=p_{K}=\frac{1}{K} P_{\text {total }}$. Then, the operator has no incentive to distinguish between the users and treats all the users equally. However, in the above system, all the users are orthogonal to each other and is a very special case of general optimization problems encountered in wireless systems.

We shall return to this problem and formally characterize the property of a central controller having no incentive to distinguish between the users as an anonymity among the users and call it interference coordination fairness in Definition 5. Now consider the following optimization problem, where the system of users cannot be orthogonalized as shown in the Example 2.

Example 3. Consider the following problem:

$$
\max _{\sum_{k \in \mathcal{K}} p_{k} \leq P_{\text {total }}} \sum_{k=1}^{K} \log \left(1+\frac{p_{k}}{\mathcal{I}_{k}(\boldsymbol{p})}\right),
$$

where $\sum_{k \in \mathcal{K}} p_{k} \leq P_{\text {total }}$ is the case of a system with a certain total power constraint of $P_{\text {total }}$. Assuming that the central controller can accurately estimate the interference at all the users and for fixed powers, we look at the following problem

$$
\max _{\sum_{k \in \mathcal{K}} p_{k} \leq P_{\text {total }}} \sum_{k=1}^{K} \log \left(1+\frac{p_{\pi(k)}}{\mathcal{I}_{k}(\boldsymbol{p})}\right),
$$

for a certain permutation $\pi$ on the set of users $\mathcal{K}$. It can be seen that in general the solution of (2) is not equivalent to the solution of (3).

The symmetric NBS, $\Phi_{N B S}(\boldsymbol{\Gamma}), \boldsymbol{\Gamma} \in \mathcal{S}^{K}$ can be obtained by maximizing the product of the utilities as follows: $\Phi_{N B S}(\boldsymbol{\Gamma})=\arg \max _{\boldsymbol{\gamma} \in \boldsymbol{\Gamma}} \prod_{k=1}^{K} \gamma_{k}$. This product optimization approach is equivalent to proportional fairness [2]. We now present certain properties of the utility function which we shall utilize in our analysis in Section IV.

Definition 3. Quasi-concavity: A function $u: \mathbb{R}_{+}^{K} \mapsto \mathbb{R}_{+}$, with $\Gamma \in \mathcal{S}_{\text {convex }}^{K}$ is quasi-concave, if and only if $\gamma_{1}, \gamma_{2} \in \boldsymbol{\Gamma}$, $\gamma_{1} \neq \gamma_{2}, u\left(\gamma_{1}\right)>u\left(\gamma_{2}\right)$, then for $\theta \in(0,1)$ we have that $u\left(\theta \gamma_{1}+(1-\theta) \gamma_{2}\right) \geq u\left(\gamma_{2}\right)$.

Definition 4. Strict monotonicity: A function $u: \mathbb{R}_{+}^{K} \mapsto \mathbb{R}_{+}$, with $\Gamma \in \mathcal{S}_{\text {convex }}^{K}$ is said to satisfy strict monotonicity, if and only if for all $\gamma_{1}, \gamma_{2} \in \Gamma, \gamma_{1} \geq \gamma_{2}$ and $\gamma_{1} \neq \gamma_{2}$ we have that $u\left(\gamma_{1}\right)>u\left(\gamma_{2}\right)$.

In the following definitions, we make an abstraction of the static state of the system and assume that the operator (central controller) has full knowledge of the interference values at the users. 
Definition 5. Axiom of equal priority: A strictly monotonic, continuous and quasi-concave function $u: \Gamma \mapsto \mathbb{R}_{+}$, for all $\boldsymbol{\Gamma} \in \mathcal{S}_{\text {convex }}^{K}$ is said to satisfy the axiom of equal priority if and only if for all permutations $\pi$, we have that $u(\gamma)=u(\pi(\gamma))$, where $\pi$ is a certain permutation over the set of users $\mathcal{K}$.

We now present the axiom of equal priority in the power domain and interference domain respectively.

Definition 6. Axiom of equal priority in the power domain: A strictly monotonic, continuous and quasi-concave function $u: \boldsymbol{\Gamma} \mapsto \mathbb{R}_{+}$, where $\gamma_{k}=p_{k} / \mathcal{I}_{k}$ is said to satisfy the axiom of equal priority in the power domain if

$$
u\left(\frac{p_{1}}{\mathcal{I}_{1}}, \ldots, \frac{p_{K}}{\mathcal{I}_{K}}\right)=u\left(\frac{p_{\pi(1)}}{\mathcal{I}_{1}}, \ldots, \frac{p_{\pi(K)}}{\mathcal{I}_{K}}\right),
$$

for all permutations $\pi$ on the set of users $\mathcal{K}$.

In Definition 6, in addition to the fact that the interference values at the users are known to the operator, it has been assumed that the interference at each user is fixed. If a utility function satisfies the axiom of equal priority in the power domain, then the operator has no priority among the various users, i.e. the operator does not choose one user over another while carrying out interference coordination or interference management so as to optimize a global objective. Example 2 does not satisfy the axiom of equal priority in the power domain. However, the example displaying proportional fairness satisfies the axiom of equal priority in the power domain

Definition 7. Axiom of equal priority in the interference domain: A strictly monotonic, continuous and quasi-concave function $u: \Gamma \mapsto \mathbb{R}_{+}$, where $\gamma_{k}=p_{k} / \mathcal{I}_{k}$ is said to satisfy the axiom of equal priority in the interference domain if

$$
u\left(\frac{p_{1}}{\mathcal{I}_{1}}, \ldots, \frac{p_{K}}{\mathcal{I}_{K}}\right)=u\left(\frac{p_{1}}{\mathcal{I}_{\pi(1)}}, \ldots, \frac{p_{K}}{\mathcal{I}_{\pi(K)}}\right),
$$

for all permutations $\pi$ on the set of users $\mathcal{K}$.

In Definition 7, in addition to the fact that the interference values at the users are known to the operator, it has been assumed that the power of each user is fixed. The axiom of equal priority in the power domain or the axiom of equal priority in the interference domain does not imply that the channel has no impact on the resource allocation. Even if each user is allocated equal power, the channel coefficients of the users decide the performance or utility obtained by that particular user. In Section IV we shall show that the axiom of equal priority in the power domain and the axiom of equal priority in the interference domain are equivalent under further conditions on the utility functions

\section{B. Axiomatic Framework}

Here we describe certain axioms, which are important in this paper.

WPO Weak Pareto Optimality: For $\boldsymbol{\Gamma} \in \mathbb{R}_{+}^{K}$, let $W(\boldsymbol{\Gamma}):=$ $\left\{\gamma^{(1)} \in \boldsymbol{\Gamma}\right.$ : there is no $\gamma^{(2)} \in \boldsymbol{\Gamma}$ with $\left.\gamma^{(2)}>\gamma^{(1)}\right\}$. Then if for every $\boldsymbol{\Gamma} \in \mathcal{S}^{K}, \Phi(\boldsymbol{\Gamma}) \in W(\boldsymbol{\Gamma}), \Phi$ satisfies $W P O$.

PO Pareto Optimality: For every $\boldsymbol{\Gamma} \in \mathcal{S}^{K}, \Phi(\boldsymbol{\Gamma}) \in$ $P(\boldsymbol{\Gamma})$, where $P(\boldsymbol{\Gamma})$ is the Pareto Optimal set defined as follows: $P(\boldsymbol{\Gamma}):=\left\{\boldsymbol{\gamma}^{(1)} \in \boldsymbol{\Gamma}: \nexists \boldsymbol{\gamma}^{(2)} \in \boldsymbol{\Gamma}\right.$ with $\left.\gamma^{(2)} \geq \gamma^{(1)}, \gamma^{(2)} \neq \gamma^{(1)}\right\}$.

II A Independence of Irrelevant Alternatives: For all $\boldsymbol{\Gamma}_{1}$, $\boldsymbol{\Gamma}_{2} \in \mathcal{S}^{K}$ with $\boldsymbol{\Gamma}_{1} \subset \boldsymbol{\Gamma}_{2}$ and $\Phi\left(\boldsymbol{\Gamma}_{2}\right) \in \boldsymbol{\Gamma}_{1}$, we have $\Phi\left(\boldsymbol{\Gamma}_{1}\right)=\Phi\left(\boldsymbol{\Gamma}_{2}\right)$.

SYM Symmetry: For every $\boldsymbol{\Gamma} \in \mathcal{S}^{K}$, if $\boldsymbol{\Gamma}$ is symmetric set then, $\Phi_{1}(\boldsymbol{\Gamma})=\Phi_{2}(\boldsymbol{\Gamma})=\ldots=\Phi_{K}(\boldsymbol{\Gamma})$, where $\Phi(\boldsymbol{\Gamma})=\left[\Phi_{1}(\boldsymbol{\Gamma}), \ldots, \Phi_{K}(\boldsymbol{\Gamma})\right]$.

STC Scale Transformation Covariance ${ }^{5}$ STC: For every $\boldsymbol{\Gamma} \in \mathcal{S}^{K}$, and all $\boldsymbol{a} \in \mathbb{R}^{K}, \boldsymbol{a}>\mathbf{0}$ and $(\boldsymbol{a} \boldsymbol{\Gamma}) \in \mathcal{S}^{K}$, we have $\Phi(\boldsymbol{a} \boldsymbol{\Gamma})=\boldsymbol{a} \Phi(\boldsymbol{\Gamma})$.

The interpretation of $W P O$ is that it is impossible to find another point, which leads to strictly superior performance for all the users in the system simultaneously. From a wireless systems perspective: when a CCF representing a resource allocation strategy satisfies the axiom of $W P O$ it implies that the operating point of the resource allocation strategy will be on the boundary of the region. In general we would always like to have an operating point on the boundary, so that we are fully utilizing all the resource.

Based on the system objective, $\Phi$ could satisfy certain other axioms, selecting one point on the boundary, which is the desired operating point. The axiom IIA implies that if the feasible set shrinks and the operating point still remains feasible, then the operating point of the smaller set should be the same.

The axiom of $S Y M$ implies that if the feasible utility set is symmetric, then the resource allocation strategy divides the resources symmetrically among the users. In wireless systems, the axiom of $S Y M$ ensures a certain kind of equity among the various users in the wireless system.

The axiom of $S T C$ implies that the solution outcome is invariant with respect to component-wise scaling of the feasible utility region. It requires that the solution outcome be independent of the chosen preferences of the users in a wireless system. We present an example below which helps clarify the axiom of $S T C$ from a wireless system perspective.

Example 4. An example of a resource allocation strategy, which satisfies the axiom of $S T C$ is as follows: multiuser SISO downlink with a sum power constraint. If the basestation now has larger power available to it, then the region scales linearly as compared to the previous case. If the solution outcome in the latter case is a linear scaling of the solution outcome in the previous case, then the corresponding resource allocation strategy is said to satisfy the axiom of STC.

The axioms described above serve as our axiomatic framework. The axioms of WPO,SYM,IIA and STC can be used to obtain a symmetric NBS for convex sets. If the utility set $\Gamma$ is closed, bounded, convex comprehensive, then we obtain an unique symmetric NBS fulfilling these four axioms.

\section{Proportional Fairness: AnONymity of Users}

In this section we present further nice characteristics of the proportional fair resource allocation strategy and motivate the

\footnotetext{
${ }^{5}$ Here we use the notation $a \Gamma=\{\gamma: \exists s \in \Gamma$ with $\gamma=a s\}$, where $a s$ is defined as component-wise multiplication.
} 
concept of interference coordination fairness. The combinatorial structure of the proportional fairness resource allocation strategy was briefly discussed in Section 3.2 in [13]. Here, we have performed the analysis with respect to the axiom of equal priority in the power domain. The same analysis, with respect to the axiom of equal priority in the interference domain can be easily carried out. The main motivation of such an analysis, as previously mentioned - is to analyze the special property, which proportional fairness inherits from its structure. The property does not allow an operator or a central controller to have a preference amongst the users in a certain sense. We call this anonymity of the users as interference coordination fairness. We now define the CCF $\Phi_{u}$ in terms of the utility function $u$ as follows:

$$
\Phi_{u}(\boldsymbol{\Gamma}):=\arg \max _{\boldsymbol{\gamma} \in \Gamma} u(\gamma) .
$$

We now present certain facts, which connect the properties of the utility function $u$ to the axioms satisfied by the corresponding $\mathrm{CCF} \Phi_{u}$.

Fact 1. Let u be a quasi-concave, continuous and strictly monotonic utility function. Then, we have that the corresponding CCF $\Phi_{u}$, defined in (4) satisfies the axioms of $P O$ and II A on the family of sets $\mathcal{S}_{\text {convex. }}^{K}$

$I I A$ is a necessary condition, however not a sufficient condition for a CCF to be expressed according to (4). The satisfaction of the axioms of $P O$ and $I I A$ along with the axiom of equal priority in the power domain will help us prove that the CCF $\Phi_{u}$ corresponds to the symmetric NBS on the family of sets $\mathcal{S}_{\text {convex }}^{K}$.

Lemma 1. Let a utility function u satisfy the axiom of equal priority in the power domain. Then, the corresponding CCF $\Phi_{u}$, defined in (4) satisfies the axiom of $S Y M$ on the family of sets $\mathcal{S}_{\text {convex }}^{K}$.

Let a utility function $u$ satisfy the axiom of equal priority in the interference domain. Then, the corresponding $C C F \Phi_{u}$, defined in (4) satisfies the axiom of SYM on the family of sets $\mathcal{S}_{\text {convex }}^{K}$.

Proof: $u\left(\gamma_{1}, \ldots, \gamma_{K}\right)=u\left(\frac{p_{1}}{\mathcal{I}_{1}}, \ldots, \frac{p_{K}}{\mathcal{I}_{K}}\right)$. Let $\mathcal{I}_{1}=\ldots=$ $\mathcal{I}_{K}=1$. Therefore

$$
\begin{aligned}
u\left(\frac{p_{1}}{\mathcal{I}_{1}}, \ldots, \frac{p_{K}}{\mathcal{I}_{K}}\right) & =u\left(p_{1}, \ldots, p_{K}\right) \\
& =u\left(\Pi_{k \in \mathcal{K}} p_{k}\right) \\
& =u\left(\Pi_{k \in \mathcal{K}} \gamma_{k}\right) .
\end{aligned}
$$

The second equality above follows from the axiom of equal priority in the power domain. The third equality follows, since the axiom of equal priority in the power domain holds for all values of fixed interference. Hence, we have proved, that if the axiom of equal priority in the power domain is fulfilled then so is the axiom of equal priority, which is equivalent to the axiom of $S Y M$.

With Lemma 1 we are able to prove the following characterization of proportional fairness.

Theorem 1. Let u be a quasi-concave, continuous and strictly monotonic utility function. Then, u satisfies the axiom of equal priority in the power domain, if and only if for all powers $p_{1}, \ldots, p_{K}>0$ and interference values $\mathcal{I}_{1}, \ldots, \mathcal{I}_{K}>0$, $u\left(\frac{p_{1}}{\mathcal{I}_{1}}, \ldots, \frac{p_{K}}{\mathcal{I}_{K}}\right)=u\left(\frac{p_{1} \ldots p_{K}}{\mathcal{I}_{1} \ldots \mathcal{I}_{K}}, 1, \ldots, 1\right)$, i.e. there exists $a$ continuous and strictly monotonic increasing function $h$, such that $u$ can be written as $u\left(\gamma_{1}, \ldots, \gamma_{K}\right):=h\left(\prod_{k \in \mathcal{K}} \gamma_{k}\right)$.

Proof: " $\Longrightarrow$ : The proof will be achieved via induction. We begin with the case for 2 users. For any $p_{1}, p_{2}, \mathcal{I}_{1}, \mathcal{I}_{2}>0$ and for any $\lambda>0$, from the axiom of equal priority in the power domain and $\mathcal{I}_{1}^{\prime}=\mathcal{I}_{1} / \lambda$ we have that

$$
u\left(\frac{\lambda p_{1}}{\mathcal{I}_{1}}, \frac{p_{2}}{\mathcal{I}_{2}}\right)=u\left(\frac{\lambda p_{2}}{\mathcal{I}_{1}}, \frac{p_{1}}{\mathcal{I}_{2}}\right) .
$$

Let $p_{2}^{\prime}=\lambda p_{2}$. Then, from the axiom of equal priority in the power domain

$$
u\left(\frac{p_{2}^{\prime}}{\mathcal{I}_{1}}, \frac{p_{1}}{\mathcal{I}_{2}}\right)=u\left(\frac{p_{1}}{\mathcal{I}_{1}}, \frac{p_{2}^{\prime}}{\mathcal{I}_{2}}\right)
$$

Therefore,

$$
u\left(\frac{\lambda p_{2}}{\mathcal{I}_{1}}, \frac{p_{1}}{\mathcal{I}_{2}}\right)=u\left(\frac{p_{1}}{\mathcal{I}_{1}}, \frac{\lambda p_{2}}{\mathcal{I}_{2}}\right)
$$

From (5) and (6) we have that

$$
u\left(\frac{\lambda p_{1}}{\mathcal{I}_{1}}, \frac{p_{2}}{\mathcal{I}_{2}}\right)=u\left(\frac{p_{1}}{\mathcal{I}_{1}}, \frac{\lambda p_{2}}{\mathcal{I}_{2}}\right)
$$

Equation (7) is valid for all $\lambda>0$.

We define $\hat{\lambda}:=\mathcal{I}_{2} / p_{2}$. Then, we have that

$$
u\left(\frac{\mathcal{I}_{2}}{p_{2}} \frac{p_{1}}{\mathcal{I}_{1}}, \frac{p_{2}}{\mathcal{I}_{2}}\right)=u\left(\frac{p_{1}}{\mathcal{I}_{1}}, 1\right) .
$$

For any $\tilde{p}_{1}, \tilde{\mathcal{I}}_{1}>0$, we define $p_{1}=\tilde{p}_{1} p_{2}$ and $\mathcal{I}_{1}=\tilde{\mathcal{I}}_{1} \mathcal{I}_{2}$. Then we have that

$$
u\left(\frac{\mathcal{I}_{2}}{p_{2}} \frac{\tilde{p}_{1} p_{2}}{\tilde{\mathcal{I}}_{1} \mathcal{I}_{2}}, \frac{p_{2}}{\mathcal{I}_{2}}\right)=u\left(\frac{\tilde{p}_{1} p_{2}}{\tilde{\mathcal{I}}_{1} \mathcal{I}_{2}}, 1\right)=u\left(\frac{\tilde{p}_{1}}{\tilde{\mathcal{I}}_{1}}, \frac{p_{2}}{\mathcal{I}_{2}}\right) .
$$

Hence, we have proved the result for the case of 2 users. Now let us assume that the result holds for the case of $K$ users. Now consider that there are $K+1$ users, with powers $p_{1}, \ldots, p_{K}, p_{K+1}>0$ and interference functions $\mathcal{I}_{1}, \ldots, \mathcal{I}_{K}, \mathcal{I}_{K+1}>0$. Then we have that

$$
u\left(\frac{p_{1}}{\mathcal{I}_{1}}, \ldots, \frac{p_{K}}{\mathcal{I}_{K}}, \frac{p_{K+1}}{\mathcal{I}_{K+1}}\right)=u\left(\frac{\prod_{l=1}^{K} p_{l}}{\prod_{l=1}^{K} \mathcal{I}_{l}}, 1, \ldots, 1, \frac{p_{K+1}}{\mathcal{I}_{K+1}}\right)
$$

where

$$
u\left(\frac{\prod_{l=1}^{K} p_{l}}{\prod_{l=1}^{K} \mathcal{I}_{l}}, 1, \ldots, 1, \frac{p_{K+1}}{\mathcal{I}_{K+1}}\right)=u\left(\frac{\hat{p}_{1}}{\hat{\mathcal{I}}_{1}}, 1, \ldots, 1, \frac{p_{K+1}}{\mathcal{I}_{K+1}}\right)
$$

Finally, from the fact, that the expression is true for the case of 2 users, we obtain

$$
u\left(\frac{\hat{p}_{1}}{\hat{\mathcal{I}}_{1}}, 1, \ldots, 1, \frac{p_{K+1}}{\mathcal{I}_{K+1}}\right)=u\left(\frac{\hat{p}_{1} p_{K+1}}{\hat{\mathcal{I}}_{1} \mathcal{I}_{K+1}}, 1, \ldots, 1\right) .
$$

Hence, we have our desired result.

"£": This direction can be easily verified. Hence, we skip the proof.

A particular example of the function $h$ is $h(x)=$ $u(x, 1, \ldots, 1)$.

Corollary 1. Let $u$ be a quasi-concave, continuous and strictly monotonic utility function. Then, $u$ satisfies the axiom of equal priority in the interference domain, if and only if for all powers $p_{1}, \ldots, p_{K}>0$ and interference values 
$\mathcal{I}_{1}, \ldots, \mathcal{I}_{K}>0, u\left(\frac{p_{1}}{\mathcal{I}_{1}}, \ldots, \frac{p_{K}}{\mathcal{I}_{K}}\right)=u\left(\frac{p_{1} \ldots p_{K}}{\mathcal{I}_{1} \ldots \mathcal{I}_{K}}, 1, \ldots, 1\right)$, i.e. there exists a continuous and strictly monotonic increasing function $h$, such that $u$ can be written as $u\left(\gamma_{1}, \ldots, \gamma_{K}\right):=$ $h\left(\prod_{k \in \mathcal{K}} \gamma_{k}\right)$.

The above results show that under certain assumptions on the utility functions the axiom of equal priority in the power domain and the axiom of equal priority in the interference domain are equivalent.

Remark 1. Let $u$ be a quasi-concave, continuous and strictly monotonic utility function. Then, the utility function $u$ satisfies the axiom of equal priority, if and only if the corresponding CCF $\Phi_{u}$, where $u\left(\gamma_{1}, \ldots, \gamma_{K}\right):=h\left(\prod_{k \in \mathcal{K}} \gamma_{k}\right)$ and $h$ is a strictly monotonic increasing and continuous function is the symmetric NBS.

\section{Discussion}

We have introduced the axiom of equal priority in the power domain (axiom of equal priority in the interference domain), which emulates the anonymity of the user identity while performing interference coordination. When the resource allocation strategy satisfies the axiom of equal priority in the power domain (interference domain), then the operator gives no preference to a particular user, while carrying out interference management. We have considered the following scenario: the central controller knows the interference values of all the users. We have seen, that if and only if the operator utilizes the proportional fair resource allocation strategy, does it have no incentive to manipulate the system (a particular user has more interference or less interference). We term the anonymity of the central controller to the identity of the users, while performing interference management or coordination as interference coordination fairness.

\section{REFERENCES}

[1] R. Mazumdar, L. G. Mason, and C. Dougligeris, "Fairness in network optimal flow control: optimality of product forms," IEEE Trans. Commun., vol. 39, no. 5, pp. 775-782, May 1991.

[2] F. P. Kelly, A. K. Maulloo, and D. K. H. Tan, "Rate control in communication networks: shadow prices, proportional fairness and stability," $J$. Operational Research Society, vol. 49, pp. 237-252, 1998.

[3] G. Caire, R. R. Mueller, and R. Knopp, "Hard fairness versus proportional fairness in wireless communications: the single-cell case," IEEE Trans. Inf. Theory, vol. 53, no. 4, pp. 1366-1385, Apr. 2007.

[4] K. Kar, S. Sarkar, and L. Tassiulas, "Achieving proportional fairness using local information in Aloha networks," IEEE Trans. Automatic Control, vol. 49, no. 10, pp. 1858-1862, Oct. 2004.

[5] X. Wang and K. Kar, "Cross-layer rate optimization for proportional fairness in multihop wireless networks with random access," IEEE $J$. Sel. Areas Commun., vol. 24, no. 8, pp. 1548-1559, Aug. 2006.

[6] M. Kaneko, P. Popovski, and J. Dahl, "Proportional fairness in multicarrier system with multi-slot frames: upper bound and user multiplexing algorithms," IEEE Trans. Wireless Commun., vol. 7, no. 1, pp. 22-26, Jan. 2008.
[7] [Online]. Available: http://www.statslab.cam.ac.uk/ frank/pf/

[8] R. D. Yates, "A framework for uplink power control in cellular radio systems," IEEE J. Sel. Areas Commun., 1995.

[9] S. Ulukus and R. Yates, "Adaptive power control and MMSE interference suppression," ACM Wireless Netw., vol. 4, no. 6, pp. 489-496, 1998.

[10] M. Schubert and H. Boche, "QoS-based resource allocation and transceiver optimization," Foundations and Trends in Communications and Information Theory, 2005/2006, vol. 2, no. 6, pp. 383-529.

[11] D. Tse and P. Viswanath, Fundamentals of Wireless Communication. Cambridge University Press, 2005.

[12] J. G. Proakis and M. Salehi, Digital Communications, 5th edition. McGraw-Hill, 2008.

[13] H. Boche and M. Schubert, "Nash bargaining and proportional fairness for wireless systems," IEEE/ACM Trans. Networking, vol. 5, pp. 14531466, Oct. 2009.

[14] G. Foschini and Z. Miljanic, "A simple distributed autonomous power control algorithm and its convergence," IEEE Trans. Veh. Technol., vol. 42, no. 4, pp. 641-646, Nov. 1993.

[15] M. Mariotti, "Collective choice functions on non-convex problems," Economic Theory, vol. 16, pp. 457-463, 2000.

[16] H. J. M. Peters, Axiomatic Bargaining Game Theory. Kluwer Academic Publishers, 1992

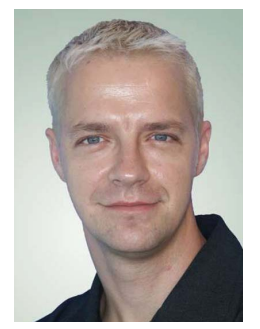

Holger Boche (M'04 - SM'07) received his Dipl.Ing. and Dr.-Ing. degrees in Electrical Engineering from the Technische Universitaet Dresden, Germany, in 1990 and 1994, respectively. In 1992 he graduated in Mathematics from the Technische Uni-versitaet Dresden, and in 1998 he received his Dr.rer.nat. degree in pure mathemat-ics from the Technische Universitaet Berlin. From 1994 to 1997, he did postgraduate studies in mathematics at the Friedrich-Schiller Universität Jena, Germany. In 1997, he joined the Heinrich-Hertz-Institut (HHI) für Nachrichtentechnik Berlin. Since 2002, he has been Full Professor for Mobile Communication Networks at the Technische Universität Berlin at the Institute for Communications Systems. In 2003, he became Director of the Fraunhofer German-Sino Lab for Mobile Communications, Berlin, Germany, and since 2004 he has also been Director of the Fraunhofer Institute for Telecommunications (HHI), Berlin, Germany. He was Visiting Professor at the ETH Zurich during winter term 2004 and 2006 and at KTH Stockholm during summer term 2005. Prof. Boche received the Research Award "Technische Kommunikation" from the Alcatel SEL Foundation in October 2003, the "Innovation Award" from the Vodafone Foundation in June 2006, Gottfried Wilhelm Leibniz Prize from the Deutsche Forschungsgemeinschaft (German Research Foundation) in 2008. He was co-recipient of the 2006 IEEE Signal Processing Society Best Paper Award and recipient of the 2007 IEEE Signal Processing Society Best Paper Award. He is a member of IEEE Signal Processing Society SPCOM and SPTM Technical Committee. He was elected a member of the German Academy of Sciences (Leopoldina) in 2008 and of Berlin Brandenburg Academy of Sciences and Humanities in 2009.

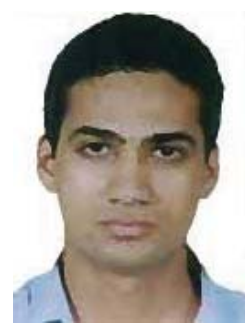

Siddharth Naik received his MS in Electrical Engineering from the Royal Institute of Technology in 2004. He was a research engineer at the SPANN laboratory at IIT, Mumbai in 2004/2005. He has been a research associate at the Heinrich Hertz Institute and has been pursuing his $\mathrm{PhD}$ in Electrical Engineering at the Technical University of Berlin, since 2006. His research interests are game theory, mechanism design, probability theory and convex optimization. 